\title{
Linearizability Is Not Always a Safety Property
}

\author{
Rachid Guerraoui $^{1}$ and Eric Ruppert ${ }^{2(\otimes)}$ \\ 1 EPFL, Lausanne, Switzerland \\ 2 York University, Toronto, Canada \\ ruppert@cse. yorku.ca
}

\begin{abstract}
We show that, in contrast to the general belief in the distributed computing community, linearizability, the celebrated consistency property, is not always a safety property. More specifically, we give an object for which it is possible to have an infinite history that is not linearizable, even though every finite prefix of the history is linearizable. The object we consider as a counterexample has infinite nondeterminism. We show, however, that if we restrict attention to objects with finite nondeterminism, we can use König's lemma to prove that linearizability is indeed a safety property. In the same vein, we show that the backward simulation technique, which is a classical technique to prove linearizability, is not sound for arbitrary types, but is sound for types with finite nondeterminism.
\end{abstract}

\section{Introduction}

One of the most challenging problems in concurrent and distributed systems is to build software objects that appear to processes using them as "perfect": always available and consistent. In particular, proving that implementations of such objects are correct can be very difficult.

To make the challenge more tractable, it is common to divide the difficulty into proving two properties: a safety and a liveness property [2,3]. In short, a safety property says "bad things should never happen" whereas a liveness property says "good things should eventually happen." Traditionally, in the context of building "perfect" shared objects, safety has been associated with the concept of linearizability [9] while liveness has been associated with a progress guarantee such as wait-freedom [8].

- Linearizability: Despite concurrent accesses to an object, the operations issued on that object should appear as if they are executed sequentially. In other words, each operation op on an object $X$ should appear to take effect at some indivisible instant between the invocation and the response of $o p$. This property, also called atomicity, transforms the difficult problem of reasoning about a concurrent system into the simpler problem of reasoning about one where the processes access each object one after another.

- Wait-freedom: No process $p$ ever prevents any other process $q$ from making progress when $q$ executes an operation on any shared object $X$. This means

(C) Springer International Publishing Switzerland 2014

G. Noubir and M. Raynal (Eds.): NETYS 2014, LNCS 8593, pp. 57-69, 2014.

DOI: $10.1007 / 978-3-319-09581-3 \_5$ 
that, provided it remains alive and kicking, $q$ completes its operation on $X$ regardless of the speed or even the failure of any other process $p$. Process $p$ could be very fast and might be permanently accessing shared object $X$, or could have failed or been swapped out by the operating system while accessing $X$. None of these situations should prevent $q$ from executing its operation.

Ensuring each of linearizability or wait-freedom alone is simple. The challenge is to ensure both. In particular, one could easily ensure linearizability using locks and mutual exclusion. But this would not guarantee wait-freedom: a process that holds a lock and fails can prevent others from progressing. One could also forget about linearizability and ensure wait-freedom by creating copies of the object that never synchronize: this would lead to different objects, one per process, defeating the sense of a shared object. So indeed the challenge is to design shared abstractions that ensure both linearizability and wait-freedom. But proving correctness can be made simpler if we could prove each property separately.

It was shown that properties of distributed systems can be divided into safety and liveness properties [2], each requiring specific kinds of proof techniques. So the general approach in proving the correctness of shared objects is that linearizability, being a safety property, requires techniques to reason about finite executions (such as backward simulation [13]), whereas wait-freedom, being a liveness property, requires another set of techniques to reason about infinite executions. The association between safety and linearizability on the one hand, and liveness and wait-freedom on the other, is considered a pillar in the theory of distributed computing.

This paper shows that, strictly speaking, this association is wrong for the most general definition of object type specifications. More specifically, we show that, in contrast to what is largely believed in the distributed computing literature, linearizability is not a safety property. This might be surprising because (a) it was often argued that linearizability is a safety property, e.g., in [12], and (b) linearizability proofs have used techniques specific to safety properties, e.g., backward simulation [13]. In fact, there is no real contradiction with our new result for the following reasons.

- To prove that linearizability is not a safety property, we exhibit an object, which we call the countdown object, and a non-linearizable history such that every finite prefix of the execution is linearizable. The object we consider has infinite nondeterminism, which might occur, for instance, in a distributed system that seeks to ensure fairness (as we discuss in Sect. 2.2). Interestingly, the execution we use in our proof is by a single process, so it demonstrates that other consistency conditions that are weaker than linearizability (such as sequential consistency) are also not safety properties for the countdown object.

- We show, however, that if we restrict attention to objects with finite nondeterminism, we can use König's lemma [10] to prove that linearizability is indeed a safety property. We thus highlight that, even if this was not always stated in 
the past, claims that linearizability is a safety property, should assume finite nondeterminism. ${ }^{1}$ Lynch's proof that linearizability is a safety property [12] applies only to the more restricted class of deterministic objects.

In the same vein, we show that the backward simulation technique, which is sometimes used to prove linearizability, is not sound for arbitrary types (if infinite nondeterminism is permitted). It is sound, however, for finite nondeterminism.

The rest of the paper is organized as follows. We describe our system model in Sect. 2. We recall the notion of linearizability in Sect. 3. In Sect. 4 we recall the concept of safety and give our counterexample that shows linearizability is not a safety property. Then we show in Sect. 5 that, if we restrict ourselves to objects with finite nondeterminism, linearizability becomes a safety property. We consider the implications for backward simulations in Sect. 6 and conclude the paper in Sect. 7 .

\section{System Model}

We consider a system consisting of a finite set of $n$ processes, denoted $p_{1}, \ldots, p_{n}$. Processes communicate by executing operations on shared objects. The execution of an operation $o p$ on an object $X$ by a process $p_{i}$ is modelled by two events, the invocation event denoted $i n v\left[X . o p\right.$ by $\left.p_{i}\right]$ that occurs when $p_{i}$ invokes the operation, and the response event denoted resp $\left[X\right.$.res by $\left.p_{i}\right]$ that occurs when the operation terminates and returns the response res. (When there is no ambiguity, we talk about operations where we should be talking about operation executions.)

\section{$2.1 \quad$ Objects}

An object has a unique identity and a type. Multiple objects can be of the same type. A type is defined by a sequential specification that consists of

- the set $Q$ of possible states for an object of that type,

- the initial state $q_{0} \in Q$,

- a set $O P S$ of operations that can be applied to the object,

- a set $R E S$ of possible responses the object can return, and

- a transition relation $\delta \subseteq Q \times O P S \times R E S \times Q$.

This specification describes how the object behaves if it is accessed by one operation at a time. If $\left(q, o p, r e s, q^{\prime}\right) \in \delta$, it means that a possible result of applying operation $o p$ to an object in state $q$ is that the object moves to state $q^{\prime}$ and returns the response res to the process that invoked op.

\footnotetext{
${ }^{1}$ For example, an erroneous claim is made in two recent papers $[1,11]$ that explicitly permit nondeterministic objects and make no restriction that the nondeterminism of the objects should be finite. The latter paper states that "linearizability is a safety property, so its violation can be detected with a finite prefix of an execution history." Using the definitions given in that paper, this statement is false. However, this does not affect the correctness of that paper's main results because those results are about objects with finite nondeterminism.
} 


\subsection{Infinite Nondeterminism}

- We say that an object is deterministic if, for all $q \in Q$ and $o p \in O P S$, there is at most one pair $\left(r e s, q^{\prime}\right)$ such that $\left(q, o p, r e s, q^{\prime}\right)$ is in the object's transition relation $\delta$.

- An object has finite nondeterminism if, for all $q \in Q$ and $o p \in O P S$, the set of possible outcomes $\left\{\left(\right.\right.$ res,$\left.\left.q^{\prime}\right):\left(q, o p, r e s, q^{\prime}\right) \in \delta\right\}$ is finite.

Dijkstra [6] argued that infinite nondeterminism should not arise in computing systems. He showed, for example, the functionality of nondeterministically choosing an arbitrary positive integer cannot be implemented in a reasonable sequential programming language. Nevertheless, there is a significant literature on infinite nondeterminism. For example, Apt and Plotkin [4] observed that infinite nondeterminism can arise naturally in systems that guarantee fairness. Consider a system of two processes $P$ and $Q$ programmed as follows, using a shared boolean variable Stop that is initially false.

$\begin{array}{ll}\text { Process } P: & \text { Process } Q: \\ \text { Stop }:=\text { true } & x:=1 \\ & \text { do until Stop } \\ & x:=x+1 \\ & \text { end do } \\ & \text { print } x\end{array}$

If these processes are run in a fair environment, where each process is guaranteed to be given infinitely many opportunities to take a step (but there is no bound on the relative speeds of the processes), $Q$ will choose and print an arbitrary positive integer. Thus, at the right level of abstraction, this system implements a choice with infinite nondeterminism. In the context of sharedmemory computing, objects with infinite nondeterminism have also occasionally arisen (e.g., [14]).

\subsection{Histories}

A (finite or infinite) sequence of invocation and response events is called a history and this is how we model an execution. We assume that processes are sequential: a process executes (at most) one operation at a time. Of course, the fact that processes are (individually) sequential does not preclude different processes from concurrently invoking operations on the same shared object.

The total order relation on the set of events induced by $H$ is denoted $<_{H}$. A history abstracts the real-time order in which the events occur. We assume that simultaneous (invocation or response) events do not affect one another, so that we can arbitrarily order simultaneous events.

A local history of $p_{i}$, denoted $H \mid p_{i}$, is a projection of $H$ on process $p_{i}$ : the subsequence $H$ consisting of the events generated by $p_{i}$. Two histories $H$ and $H^{\prime}$ are said to be equivalent if they have the same local histories, i.e., for each process $p_{i}, H\left|p_{i}=H^{\prime}\right| p_{i}$. 
As we are interested only in histories generated by sequential processes, we focus on histories $H$ such that, for each process $p_{i}, H \mid p_{i}$ is well-formed: it starts with an invocation, followed by a response (the matching response associated with the same object), followed by another invocation, and so on.

An operation is said to be complete in a history if the history includes both the events corresponding to the operation's invocation and its response. Otherwise, we say that the operation is pending. A history is complete if it has no pending operations and incomplete otherwise.

A history $H$ induces an irreflexive partial order on its operations as follows. Let $o p$ and $o p^{\prime}$ be two operations. Informally, operation $o p$ precedes operation $o p^{\prime}$, if $o p$ terminates before $o p^{\prime}$ starts. More precisely:

$$
\left(o p \rightarrow_{H} o p^{\prime}\right) \stackrel{\text { def }}{=}\left(\operatorname{resp}[o p]<_{H} i n v\left[o p^{\prime}\right]\right) .
$$

Two operations $o p$ and $o p^{\prime}$ are said to overlap (we also say are concurrent) in a history $H$ if neither $o p \rightarrow_{H} o p^{\prime}$ nor $o p^{\prime} \rightarrow_{H} o p$.

\subsection{Sequential Histories}

A history is sequential if its first event is an invocation, and then (1) each invocation event, except possibly the last, is immediately followed by the matching response event, and (2) each response event, except possibly the last, is immediately followed by an invocation event. A complete sequential history always ends with a response event. A history that is not sequential is said to be concurrent. Given that a sequential history $S$ has no overlapping operations, the associated partial order $\rightarrow_{S}$ defined on its operations is actually a total order.

Let $S \mid X(S$ at $X)$ denote the subsequence of history $S$ made up of all the events involving object $X$. We say that a sequential history $S$ is legal if, for each object $X$, the sequence $X . o p_{1}, X . r e s_{1}, X . o p_{2}, X . r e s_{2}, \ldots$ satisfies the sequential specification $\left(Q, q_{0}, O P S, R E S, \delta\right)$ of $X$ in the following sense: there exists $q_{1}, q_{2}, \ldots$ in $Q$ such that $\left(q_{i-1}, o p_{i}\right.$, res $\left._{i}, q_{i}\right) \in \delta$ for all $i$.

\section{$3 \quad$ Linearizability}

Linearizability [9] basically requires that each operation on an object appears to execute at some indivisible point in time, also called the operation's linearization point, between the invocation and response of the operation. Linearizability provides the illusion that the operations issued by the processes on the shared objects are executed one after another.

We first define linearizability for complete histories $H$, i.e., histories without pending operations, and then extend the definition to incomplete histories. A complete history $H$ is linearizable if there is a "witness" history $S$ such that:

1. $H$ and $S$ are equivalent,

2. $S$ is sequential and legal, and

3. $\rightarrow H \subseteq \rightarrow_{S}$. 
This means that for a history $H$ to be linearizable, there must exist a permutation $S$ of $H$, which satisfies the following requirements. First, $S$ has to be indistinguishable from $H$ to any process. Second, $S$ has to be sequential (interleaving the process histories at the granularity of complete operations) and legal (respecting the sequential specification of each object). Notice that, as $S$ is sequential, $\rightarrow_{S}$ is a total order. Finally, $S$ must also respect the real-time occurrence order of the operations as defined by $\rightarrow H$. Such a sequential history $S$ is called a linearization of $H$.

The definition of linearizability is extended to incomplete histories as follows. An incomplete history $H$ is linearizable if $H$ can be completed, i.e., modified in such a way that every invocation of a pending operation is either removed or completed with a response event, so that the resulting (complete) history $H^{\prime}$ is linearizable. Intuitively, $H^{\prime}$ is obtained by adding response events to certain pending operations of $H$, as if these operations have indeed been completed, but also by removing invocation events from some of the pending operations of $H$. We require however that all complete operations of $H$ are preserved in $H^{\prime}$.

When proving that an algorithm implements a linearizable object, we need to prove that all histories generated by the algorithm are linearizable. A history $H$ may allow for several different linearizations.

\section{Linearizability Is Not a Safety Property}

\subsection{Safety}

Intuitively, safety properties ensure that nothing "bad" ever happens. More specifically, a safety property is a set of histories that is non-empty, prefix-closed and limit-closed. Thus, a set $P$ of histories is a safety property if it satisfies the following three conditions.

- $P$ is non-empty: $P \neq\{\}$.

- $P$ is prefix-closed: if $H \in P$, then for every prefix $H^{\prime}$ of $H, H^{\prime} \in P$.

- $P$ is limit-closed: for every infinite sequence $H_{0}, H_{1}, \ldots$ of histories, where each $H_{i}$ is a prefix of $H_{i+1}$ and each $H_{i} \in P$, the limit history $H=\lim _{i \rightarrow \infty} H_{i}$ is in $P$.

To ensure that a safety property $P$ holds for a given implementation, it is thus enough to show that every finite history of the implementation is in $P$; an execution is in $P$ if and only if each of its finite prefixes is in $P$. Indeed, every infinite history of an implementation is the limit of some sequence of ever-extending finite histories and thus should also be in $P$.

\subsection{Counterexample}

Theorem 1. Linearizability is not a safety property. 


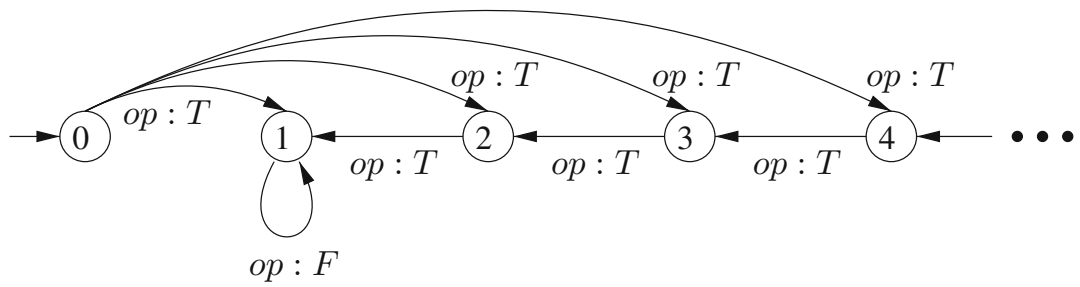

Fig. 1. The countdown object.

Proof. We define a type of object called a countdown object, which provides a single operation $o p$ that outputs $T$ or $F$. The first invocation of $o p$ nondeterministically picks a positive integer $k$. The object returns $T$ for the first $k$ invocations of op. After that, it returns $F$ for all remaining invocations of op. Formally, this type has the following sequential specification, which is illustrated in Fig. 1.

$$
\begin{aligned}
Q & =\mathbb{N} \\
q_{0} & =0 \\
O P S & =\{o p\} \\
R E S & =\{T, F\} \\
\delta & =\{(0, o p, T, k): k \geq 1\} \cup\{(1, o p, F, 1)\} \cup\{(k, o p, T, k-1): k \geq 2\}
\end{aligned}
$$

Consider the following infinite sequential history $H$ that uses a single countdown object $X$.

$$
\begin{aligned}
& i n v[X . o p \text { by } p] \\
& \operatorname{resp}[X . T \text { by } p], \\
& i n v[X . o p \text { by } p], \\
& \operatorname{resp}[X . T \text { by } p] \\
& \operatorname{inv}[X . o p \text { by } p] \\
& \operatorname{resp}[X . T \text { by } p]
\end{aligned}
$$

We first show that this history is not legal (and hence not linearizable). If we try to assign any positive integer state $k$ to the object $X$ after the first operation has been performed, then the states of the object after the next $k-1$ operations must be $k-1, k-2, k-3, \ldots, 1$. Thus, the $(k+1)$ th invocation of op in the execution would have to return $F$. Since there is no way to assign states to the object consistent with all responses in $H$, we conclude that $H$ is not legal.

Now consider any finite prefix $H^{\prime}$ of $H$. We show that $H^{\prime}$ is legal (and hence linearizable). Let $k$ be the number of complete operations in $H^{\prime}$. We can assign the sequence of states $k, k-1, \ldots, 2,1$ to $X$. Note that $(0, o p, T, k)$ and $(i, o p, T, i-1$ ) (for $2 \leq i \leq k$ ) are transitions of a countdown type, so this sequence of states satisfies the definition of legality for $H^{\prime}$. 
Let $H_{i}$ be the prefix of $H$ consisting of the first $i$ complete operations. Then, for all $i, H_{i}$ is linearizable and $H_{i}$ is a prefix of $H_{i+1}$. However, $H=\lim _{i \rightarrow \infty} H_{i}$ is not linearizable. Thus, the property of being linearizable is not limit-closed, and linearizability is not a safety property for this object specification.

Remark 2. Because the execution used in the proof of Theorem 1 is a sequential execution, the argument in fact shows that even legality is not a safety property for the countdown object type, since the sequential execution is not legal but every prefix of it is. Moreover, since the execution in the proof is by a single process, it also demonstrates that other consistency conditions that are weaker than linearizability (such as sequential consistency) are also not safety properties for the countdown object.

\section{$5 \quad$ When Linearizability Is a Safety Property}

We now show that a slight generalization of König's (Infinity) Lemma enables us to show that linearizability, when restricted to objects with finite nondeterminism, is a safety property. König's Lemma can be formulated as follows.

Lemma 3. (König's Lemma [10]). Let $G$ be an infinite directed graph such that (1) each vertex of $G$ has finite outdegree, (2) each vertex of $G$ is reachable from some root vertex of $G$ (a vertex with zero indegree), and (3) $G$ has only finitely many roots. Then $G$ has an infinite path with no repeated vertices starting from some root.

Theorem 4. Linearizability is a safety property for object types with finite nondeterminism.

Proof. Consider any object type with finite nondeterminism. The set of linearizable histories is non-empty, since the empty history (consisting of 0 events) is trivially linearizable. We show that the set of linearizable histories is prefix- and limit-closed.

Consider a linearizable history $H$. We show that any prefix $H^{\prime}$ of $H$ is also linearizable. Let $S$ be any linearization of $H$. Let sequential history $S^{\prime}$ be the shortest prefix of $S$ that contains all complete operations of $H^{\prime}$.

We claim that $S^{\prime}$ is a linearization of $H^{\prime}$. We complete $H^{\prime}$ by appending responses that are present in $S^{\prime}$ but not in $H^{\prime}$ to the end of $H^{\prime}$ and removing operations that do not appear in $S^{\prime}$. Note that only incomplete operations are removed from $H^{\prime}$ since all complete ones appear in $S^{\prime}$. Let $\bar{H}^{\prime}$ denote the resulting complete history.

First we show that complete histories $S^{\prime}$ and $\bar{H}^{\prime}$ contain the same set of operations. Any operation in $\bar{H}^{\prime}$ must also be in $S^{\prime}$ (since all operations not in $S^{\prime}$ are removed when forming $\bar{H}^{\prime}$ ). To derive a contradiction, suppose that $S^{\prime}$ contains an operation op that does not appear in $\bar{H}^{\prime}$. Since only operations that do not appear in $S^{\prime}$ were removed from $H^{\prime}$ to obtain $\bar{H}^{\prime}$, op does not appear in $H^{\prime}$ either. Since $S^{\prime}$ is the shortest prefix of $S$ that contains all complete operations 
of $H^{\prime}$, the last operation $o p^{\prime}$ in $S^{\prime}$ must be a complete operation in $H^{\prime}$. Thus, $o p \neq o p^{\prime}$. Since $o p^{\prime}$ is complete in $H^{\prime}$ and $o p$ does not appear in $H^{\prime}, o p^{\prime}<_{H} o p$. But $o p<_{S} o p^{\prime}$, contradicting the assumption that $S$ is a linearization of $H$.

Since $S^{\prime}$ is a prefix of a legal history $S$, it is also legal. Moreover, it also respects the real-time order in $\bar{H}^{\prime}$ : if $o p<_{\bar{H}^{\prime}} o p^{\prime}$, then $o p<_{S^{\prime}} o p^{\prime}$ (otherwise, $S$ would violate the real-time order in $H$ ). Since $S$ and $\bar{H}^{\prime}$ contain the same set of operations, $S^{\prime}$ respects the real-time order of $\bar{H}^{\prime}$, and local histories are well-formed, $S^{\prime}$ is equivalent to $\bar{H}^{\prime}$ : local histories in $S^{\prime}$ and $\bar{H}^{\prime}$ are identical.

So, $S^{\prime}$ is a linearization of $H^{\prime}$ and, thus, linearizability is prefix-closed.

To prove the limit-closed property, we consider an infinite sequence of everextending linearizable histories $H_{0}, H_{1}, H_{2}, \ldots$ Our goal is to show that $H=$ $\lim _{i \rightarrow \infty} H_{i}$ is linearizable. We assume that $H_{0}$ is the empty history and each $H_{i+1}$ is a one-event extension of $H_{i}$. (By prefix-closedness, each prefix of every $H_{i}$ is linearizable, so there is no loss of generality in this assumption.)

Now we construct a directed graph $G=(V, E)$ as follows. Vertices of $G$ are all tuples $\left(H_{i}, S, W\right)$, where $i \in \mathbb{N}, S$ is any linearization of $H_{i}$ that ends with a complete operation present in $H_{i}$, and $W$ is a sequence of states that witnesses the legality of $S$. There is a directed edge $\left(\left(H_{i}, S, W\right),\left(H_{j}, S^{\prime}, W^{\prime}\right)\right)$ in $G$ if and only if $j=i+1, S$ is a prefix of $S^{\prime}$ and $W$ is a prefix of $W^{\prime}$.

Note that for each $H_{i}$ there is at least one vertex $\left(H_{i}, S, W\right)$, since $H_{i}$ is linearizable and if we remove all operations at the end of the linearization that are incomplete in $H_{i}$, we still have a linearization of $H_{i}$ (the incomplete operations can also be removed from $H_{i}$ to obtain a completion of $H_{i}$ ). Moreover, since $S$ is necessarily legal, there exists a witness $W$ for it. Thus, the graph $G$ contains infinitely many vertices.

We use König's lemma to show that the resulting graph $G$ contains an infinite path $\left(H_{0}, S_{0}, W_{0}\right),\left(H_{1}, S_{1}, W_{1}\right), \ldots$ and the limit $\lim _{i \rightarrow \infty} S_{i}$ is a linearization of the infinite limit history $H$. The legality of $\lim _{i \rightarrow \infty} S_{i}$ is witnessed by the infinite sequence of states $\lim _{i \rightarrow \infty} W_{i}$.

First we observe that for each vertex $\left(H_{i+1}, S^{\prime}, W^{\prime}\right)$ (with $i \geq 0$ ), there is an edge into the vertex from some vertex $\left(H_{i}, S, W\right)$. There are two cases to consider.

- The last operation op of $S^{\prime}$ is a complete operation in $H_{i}$. In this case, $S^{\prime}$ is also a linearization of $H_{i}$. Indeed, even if the last event of $H_{i+1}$ is the invocation of a new operation $o p^{\prime}$, this operation cannot appear in $S^{\prime}$ : it can only appear before op in $S^{\prime}$ violating the real-time order in $H_{i+1}$. Thus, $\left(H_{i}, S^{\prime}, W^{\prime}\right)$ is a vertex in $G$ and there is an edge from it to $\left(H_{i+1}, S^{\prime}, W^{\prime}\right)$.

- The last operation op of $S^{\prime}$ is not a complete operation in $H_{i}$. But since $S^{\prime}$ ends with an operation op that is complete in $H_{i+1}$ and $H_{i+1}$ extends $H_{i}$ with one event only, we conclude that the last event of $H_{i+1}$ is the response of $o p$. Thus, $H_{i}$ and $H_{i+1}$ contain the same set of operations, except that $o p$ is incomplete in $H_{i}$. Let $S$ be the longest prefix of $S^{\prime}$ that ends with a complete operation in $H_{i}$. Let $W$ be the prefix of $W^{\prime}$ whose length corresponds is the number of operations in $S^{\prime}$. Since $W$ witnesses the legality of $S, W^{\prime}$ witnesses 
the legality of $S^{\prime}$. Also, only incomplete operations in $H_{i}$ do not appear in $S$. Thus, $S$ is a linearization of $H_{i}$ and $\left(H_{i}, S, W\right)$ is a vertex in $G$ and there is an edge from it to $\left(H_{i+1}, S^{\prime}, W^{\prime}\right)$.

It follows that the graph $G$ has only one root vertex, $\left(H_{0}, S_{0}, W_{0}\right)$, where $H_{0}, S_{0}$ and $W_{0}$ are empty sequences, and moreover that every vertex is reachable from this root.

Now we show that the outdegree of every vertex of $G$ is finite. There are only finitely many operations in $H_{i+1}$ and each linearization of $H_{i+1}$ is a permutation of these operations, so there can only be finitely many linearizations $S^{\prime}$ of $H_{i+1}$. Moreover for any finite-length sequential history $S^{\prime}$ there can only be finitely many witnesses to the legality of $S^{\prime}$, since the number of possible states after any finite number of operations has been performed is finite. (This is where we use the assumption that the object's specification has finite nondeterminism.) Thus, there are only finitely many vertices of the form $\left(H_{i+1}, S^{\prime}, W^{\prime}\right)$. Since all outgoing edges of any vertex $\left(H_{i}, S, W\right)$ are directed to vertices of the form $\left(H_{i+1}, S^{\prime}, W^{\prime}\right)$, the outdegree of every such vertex is also finite.

By Lemma 3, $G$ contains an infinite path starting from the root vertex: $\left(H_{0}, S_{0}, W_{0}\right),\left(H_{1}, S_{1}, W_{1}\right), \ldots$ Let $S=\lim _{i \rightarrow \infty} S_{i}$ and $W=\lim _{i \rightarrow \infty} W_{i}$. First, note that $W$ witnesses the legality of $S$. We argue now that the $S$ is a linearization of the infinite history $H$. Let $H^{\prime}$ be the completion of $H$ obtained by removing the incomplete operations of $H$ that are not included in $S$ and inserting into $H$ response events for incomplete operations of $H$ that are included in $S$. (The response events should be inserted in the order they occur in $S$ and the response to an operation $o p$ should be inserted after the response to any operation that appears before $o p$ in $S$.) By construction, $S$ is equivalent to $H^{\prime}$, and $S$ respects the real-time order of $H$; otherwise there would be a vertex $\left(H_{i}, S_{i}\right)$ such that $S_{i}$ is not equivalent to $H_{i}$ or violates the real-time order of $H_{i}$. Thus, $S$ is indeed a linearization of $H$, which concludes the proof that linearizability is a safety property.

\section{Backward Simulations}

A backward simulation [13] is a technique that is sometimes used to show that an implementation of a shared object is linearizable (for example, [5,7]). A backward simulation from a system $A$ to a system $A^{\prime}$ (which have state sets $Q$ and $Q^{\prime}$, respectively) is a relation $b s r \subseteq Q \times Q^{\prime}$ with the following properties.

1. For every state $s$ of $A$, there is a state $s^{\prime}$ of $A^{\prime}$ such that $\left(s, s^{\prime}\right) \in b s r$.

2. If there is a transition $\alpha$ from state $s_{1}$ to state $s_{2}$ in $A$ and $\left(s_{2}, s_{2}^{\prime}\right) \in b s r$ then there is a state $s_{1}^{\prime}$ and a sequence of transitions $\alpha^{\prime}$ of $A^{\prime}$ such that $\left(s_{1}, s_{1}^{\prime}\right) \in b s r, \alpha^{\prime}$ moves from state $s_{1}^{\prime}$ to $s_{2}^{\prime}$, and the sequence of externally observable events ${ }^{2}$ is the same in $\alpha$ and $\alpha^{\prime}$.

${ }^{2}$ In the context of implementations of shared object of type $T$, observable events are just invocations and responses on the object of type $T$. 
3. If $q_{0}$ is a possible initial state of $A$ and $\left(q_{0}, q_{0}^{\prime}\right) \in b s r$ then $q_{0}^{\prime}$ is a possible initial state of $A^{\prime}$.

If such a backward simulation exists from an implementation automaton to an abstract automaton that specifies correct linearizable behaviour, it is easy to prove that every finite history of the implementation is also a history of the abstract automaton, and hence linearizable. Intuitively, given a history $H$ of the implementation $A$, we start from the final state of that history and find a matching state of the abstract automaton $A^{\prime}$ using property 1 . Then, working backwards step by step, we build a history $H^{\prime}$ of $A^{\prime}$ by finding, for each transition of $H$, a sequence of transitions to prepend to $H^{\prime}$ using property 2 . Finally, when we reach the beginning of $H$ we observe, using property 3 , that the history we have built could take place starting from an initial state of $A^{\prime}$. Moreover, by construction the two histories have the same sequence of externally visible events.

Thus, if we can build a backward simulation from the implementation to the abstract automaton that specifies linearizable behaviour and if linearizability is a safety property, it follows that the implementation is linearizable. However, if linearizability is not a safety property, then the existence of the backward simulation does not necessarily imply that the implementation is linearizable. In fact, we can provide an example of an incorrect implementation of the countdown object where there is a backward simulation between the implementation and the abstract automaton.

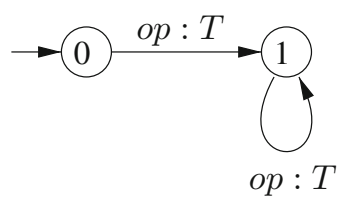

Fig. 2. An incorrect implementation of the countdown object.

Consider the following trivial (but incorrect) implementation of a countdown object: to perform an op on the countdown object, a process immediately returns $T$. One way to model this implementation is the automaton shown in Fig. 2. The reason that this implementation is incorrect is that it is possible for the implementation to return $T$ forever in an infinite execution, something that is not permitted by the specification of the countdown object. Nevertheless, there is a backward simulation relation from the implementation to the countdown type. Let

$$
b s r=\{(0,0)\} \cup\{(1, k): k \geq 1\} .
$$

It is easy to verify that $b s r$ satisfies the three properties that define a backward simulation relation using the following correspondence between actions. 


\begin{tabular}{l|l|l|l}
\hline$\alpha$ & $s_{2}^{\prime}$ & $\alpha^{\prime}$ & External actions \\
\hline $0 \rightarrow 1$ & $k$, where $k \geq 1$ & $0 \rightarrow k$ & op $: T$ \\
$1 \rightarrow 1$ & $k$, where $k \geq 1$ & $k+1 \rightarrow k$ & op $: T$ \\
\hline
\end{tabular}

Thus, for objects with infinite nondeterminism, backward simulations are not necessarily a sound technique for proving linearizability (unless one can also prove that linearizability is a safety property for the object type considered). In view of Theorem 4, proving linearizability with a backward simulations is sound for any type with finite nondeterminism.

\section{Concluding Remarks}

For clarity, we have used the terms finite nondeterminism, rather than bounded nondeterminism (which is often used in the literature) because an object may have finite nondeterminism even when there is no bound $B$ such that the number of possible responses to an operation is always bounded by $B$. For example, consider the bag object type, which stores a set of natural numbers and provides two operations: insert $(k)$, which adds $k$ to the set, and delete, which nondeterministically removes and returns an arbitrary element of the set. It has the following formal specification.

$$
\begin{aligned}
Q= & \mathcal{P}(\mathbb{N}) \\
q_{0}= & \{\} \\
O P S= & \{\operatorname{insert}(k): k \in \mathbb{N}\} \cup\{\text { delete }\} \\
R E S= & \{\text { ack, empty }\} \cup \mathbb{N} \\
\delta= & \{(S, \text { insert }(k), \text { ack, } S \cup\{k\}): S \subseteq \mathbb{N}, k \in \mathbb{N}\} \cup \\
& \{(S, \text { delete }, k, S-\{k\}): S \subseteq \mathbb{N}, k \in S\} \cup\{(\{\}, \text { delete, empty, }\{\})\}
\end{aligned}
$$

Although the number of nondeterministic choices available to a delete operation depends on the current state, and there is no a priori bound on this number, the bag object does have finite nondeterminism, so Theorem 4 says that linearizability is a safety property for the bag object.

We have shown in this paper that, strictly speaking, linearizability is not a safety property if infinite nondeterminism is permitted in the definition of object types. This points out the importance of considering carefully whether theorems proved about shared-memory systems apply to arbitrary nondeterministic object type specifications, or whether one should make the (often reasonable) restriction that object types must have finite nondeterminism. In particular, this shows that if linearizability is established by proving that every finite run is linearizable, for example by using a backward simulation, then there is an additional proof obligation to show that linearizability is a safety property for the particular type, for example by showing that the type specification has finite nondeterminism and then applying Theorem 4 of this paper. One open question raised by this 
work would be to give a precise characterization of the object types for which linearizability is a safety property.

Acknowledgements. The model section and definition of linearizability are based on lecture notes written by the first author with Michel Raynal and then with Petr Kuznetsov. The proof of Theorem 2 is inspired by a proof by Petr Kuznetsov, itself inspired by a proof by Nancy Lynch [12]. We thank Franck van Breugel for helpful discussions.

\section{References}

1. Adhikari, K., Street, J., Wang, C., Liu, Y., Zhang, S.J.: Verifying a quantitative relaxation of linearizability via refinement. In: Bartocci, E., Ramakrishnan, C.R. (eds.) SPIN 2013. LNCS, vol. 7976, pp. 24-42. Springer, Heidelberg (2013)

2. Alpern, B., Schneider, F.B.: Defining liveness. Inf. Process. Lett. 21(4), 181-185 (1985)

3. Alpern, B., Schneider, F.B.: Recognizing safety and liveness. Distrib. Comput. 2(3), 117-126 (1987)

4. Apt, K.R., Plotkin, G.D.: A cook's tour of countable nondeterminism. In: Even, S., Kariv, O. (eds.) Automata, Languages and Programming. LNCS, vol. 115, pp. 479-494. Springer, Heidelberg (1981)

5. Colvin, R., Groves, L., Luchangco, V., Moir, M.: Formal verification of a lazy concurrent list-based set algorithm. In: Ball, T., Jones, R.B. (eds.) CAV 2006. LNCS, vol. 4144, pp. 475-488. Springer, Heidelberg (2006)

6. Dijkstra, E.W.: On nondeterminacy being bounded. In: Dijkstra, E.W. (ed.) A Discipline of Programming, Chap. 9. Prentice-Hall, Englewood Cliffs (1976)

7. Doherty, S., Moir, M.: Nonblocking algorithms and backward simulation. In: Keidar, I. (ed.) DISC 2009. LNCS, vol. 5805, pp. 274-288. Springer, Heidelberg (2009)

8. Herlihy, M.P.: Wait-free synchronization. ACM Trans. Program. Lang. Syst. 13(1), 123-149 (1991)

9. Herlihy, M.P., Wing, J.M.: Linearizability: a correctness condition for concurrent objects. ACM Trans. Program. Lang. Syst. 12(3), 463-492 (1990)

10. König, D.: Über eine Schlussweise aus dem Endlichen ins Unendliche. Acta Litterarum ac Scientiarum Regiae Universitatis Hungaricae Francisco-Josephinae: Sectio Scientiarum Mathematicarum 3, 121-130 (1927). also in chapter VI of Dénes König. Theory of Finite and Infinite Graphs, Birkhäuser, Boston, 1990

11. Liu, Y., Chen, W., Liu, Y.A., Sun, J., Zhang, S.J., Dong, J.S.: Verifying linearizability via optimized refinement checking. IEEE Trans. Softw. Eng. 39(7), 1018-1039 (2013)

12. Lynch, N.: Distributed Algorithms, Chap. 13. Morgan Kaufmann, San Mateo (1996)

13. Lynch, N.A., Vaandrager, F.W.: Forward and backward simulations. Inf. Comput. 121(2), 214-233 (1995)

14. Schenk, E.: The consensus hierarchy is not robust. In: Proceedings of 16th ACM Symposium on Principles of Distributed Computing, p. 279 (1997) 